\title{
Dynamics of squeeze-out: Theory and experiments
}

\author{
S. Zilberman ${ }^{\text {a) }}$ \\ School of Chemistry, Tel Aviv University, Tel Aviv, Israel 69978 \\ T. Becker and F. Mugele \\ Universität Ulm, Abteilung Angewandte Physik, 89069 Ulm, Germany \\ B. N. J. Persson ${ }^{\text {b) }}$ \\ IFF, FZ-Jülich, 52425 Jülich, Germany
}

A. Nitzan
School of Chemistry, Tel Aviv University, Tel Aviv, Israel 69978

(Received 4 September 2002; accepted 24 March 2003)

\begin{abstract}
We consider the dynamics of squeeze-out of a molecularly thin confined two-dimensional (2D) liquidlike layer. The squeeze-out is described by a generalized 2D Navier-Stokes equation which is solved exactly for the limiting case where the squeeze-out nucleates at the center of the contact area, and where the (perpendicular) three-dimensional pressure profile is Hertzian. We also present numerical results for the case where the nucleation is off-center. The theoretical results are in good agreement with recent experimental data by two of us for octamethylcyclotetrasiloxane. In light of our theoretical model calculations, we also discuss the spatially resolved diffusion experiments of Mukhopadhyay et al. [Phys. Rev. Lett. 89, 136103 (2002)]. Here, we obtain a puzzling disagreement between theory and experiment which requires more investigation. (C) 2003 American Institute of Physics. [DOI: 10.1063/1.1574790]
\end{abstract}

\section{INTRODUCTION}

Sliding friction is one of the oldest problems in physics, and undoubtedly has a huge practical importance. ${ }^{1-3}$ In recent years, the ability to produce durable low-friction surfaces and lubricants has become an important factor in the miniaturization of moving components in technologically advanced devices. For such applications, the interest is focused on the stability under pressure of thin lubricant films, since the complete squeeze-out of the lubricant from an interface may give rise to cold-welded junctions, resulting in high friction and catastrophically large wear.

When two elastic solids with curved but atomically smooth surfaces are brought into contact in a fluid, a small circular (or eliptic) Hertzian contact area is formed, where the surfaces are parallel and separated by an integer number of monolayers of trapped lubricant fluid. Outside this contact area the solid walls are curved, and separated by a lubricant film with a thickness which increases continuously with the distance from the periphery of the contact area.

It has been shown both experimentally and theoretically that when simple fluids (quasispherical molecules and linear hydrocarbons) are confined between atomically flat surfaces at microscopic separations, the behavior of the lubricant is mainly determined by its interaction with the solids that induce layering in the perpendicular direction. ${ }^{4-11}$ The thinning of the lubrication film occurs stepwise, by expulsion of individual layers. These layering transitions appear to be thermally activated. ${ }^{12,13}$ Under strong confinement conditions, some lubricant fluids become solidlike. ${ }^{4-11}$ Other fluids, no-

\footnotetext{
${ }^{a)}$ Electronic mail: silviu@post.tau.ac.il

b) Author to whom correspondence should be addressed. Electronic mail: b.persson@fz-juelich.de
}

tably water, ${ }^{14,15}$ remain liquidlike up to the last layer that can be removed upon squeezing.

The phenomenology of layering transitions in 2D-solidlike boundary lubrication has been studied in Refs. 16 and 17. We have shown in a series of computer simulations ${ }^{18}$ that the layering transitions are sometimes initiated in solidlike layers by a disordering transition, after which the lubricant behaves in a liquidlike manner for the rest of the squeeze process. Since the typical lateral extension in surface force apparatus (SFA) experiments is of the order 10-100 $\mu \mathrm{m}$ (much greater than atomic dimensions), it is reasonable to expect that during the layering transition the squeeze-out can often be described in the framework of $2 \mathrm{D}$ continuum fluid mechanics.

Such layering transitions were recently observed for the first time by Mugele and Salmeron for a chain alcohol, $\mathrm{C}_{11} \mathrm{H}_{23} \mathrm{OH}$ (Refs. 19, 20), by imaging the lateral variation of the gap between the solid surfaces as a function of time. In those experiments they were able to study the $n=1 \rightarrow 0$ transition. More recently, in a refined experimental setup, two of us $^{21}$ were able to image several layering transitions $(n \rightarrow n$ $-1, n=5,4,3,2)$ of the silicone oil octamethylcyclotetrasiloxane (OMCTS, spherical molecule, diameter $\sim 10 \AA$ ) in great detail.

The basic theory of 2D squeeze-out dynamics was described in Ref. 12. Initially the system is trapped in a metastable state at the initial film thickness. Squeeze-out starts by a thermally activated nucleation process in which a density fluctuation forms a small hole, of critical radius $R_{c} \sim 10 \AA$. Once formed, a 2D pressure difference $\Delta p$ develops between the boundary line separating the squeezed-out region from the rest of the system, and the (circular) boundary line of the 


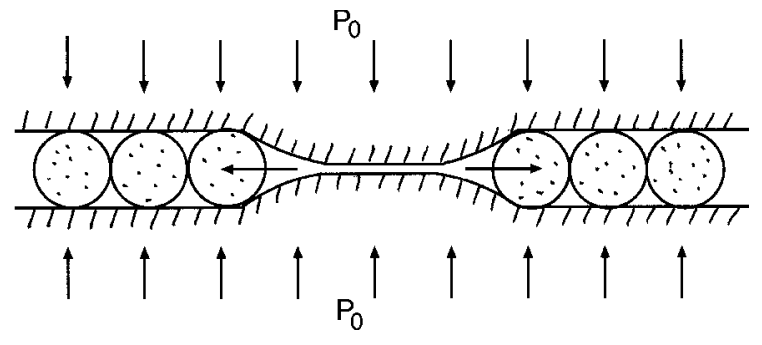

FIG. 1. Because of the curvature of the solid walls at the boundary line, the perpendicular pressure $P_{0}$ will give rise to a parallel force component acting on the 2D-lubrication film.

contact area, thus driving out the rest of the $2 \mathrm{D}$ fluid. The origin of $\Delta p$ is the elastic relaxation of the confining solids at the boundary line as is illustrated in Fig. 1.

In principle, the same reasoning of the $2 \mathrm{D}$ squeeze-out model for $n=1 \rightarrow 0$ transition applies to $n=2$ or $n=3$ (or larger $n$ ) lubricant films. Molecular dynamics computer simulations (e.g., Ref. 18) for weakly adsorbed films (physisorbed molecules) show that during squeeze-out there are no shear deformations of the film in the $z$ direction perpendicular to the interface, and slip occurs at the solid-film interfaces. Under these conditions we can treat the film as 2D during squeeze-out, and use the (modified) 2D NavierStokes equation as if the film is liquidlike in the $x-y$ plane as assumed in our numerical simulations (which agree well with the experimental data). Note that Navier-Stokes hydrodynamics are, in principle, invalid for a strict 2D (infinitely extended) fluid. We consider this, however, to be of purely academic interest. The corrections to quantities such as the viscosity depend logarithmically on the size of the physical system and are small even for macroscopic systems (the situation is similar to that of 2D elastic solids). Furthermore, it has been shown ${ }^{22}$ that the frictional coupling to the third dimension (which is included in our treatment) will regulate the theory.

It has been shown experimentally and theoretically ${ }^{19,20,23,24}$ that instabilities in the boundary line between the squeezed and nonsqueezed areas leads to a lower bound on the typical roughness wavelength $\lambda_{c}$ of the interfacial line. Another interesting experimental observation were trapped fluid pockets left behind long after the bulk of the squeeze is done. The typical size of these fluid pockets was shown to be of order $\lambda_{c}$.

The theoretical and simulation work of the present paper were stimulated by our recent measurements, which will be presented in more detail elsewhere. ${ }^{21}$ With a greatly refined experimental setup, two of us were able to image the $n \rightarrow n$ $-1(n=5,4,3,2)$ layering transitions for OMCTS confined between two flat mica surfaces. The spatial resolution was good enough to extract detailed information of the squeezeout dynamics. Here, we compare these experimental data with our hydrodynamic squeeze-out theory. We note that this is the first rigorous test of this theory. New computer simulations results are presented for the case where the line energy at the squeeze-out boundary is much higher than in the earlier studies. This leads to a smooth boundary line as observed in the new experimental presented below. The physi- cal reason for the difference in the line energy between the new $^{21}$ and older ${ }^{19,20}$ experiments is related to the difference in the thickness of the mica films (see the discussion below in Sec. IV). Under these circumstances, we show that the evolution of the boundary line separating the squeezed and nonsqueezed areas is quasicircular both in experiments and in simulations. These observations led us to derive new exact solution for a model of 2D circular squeeze-out; we calculate the radius as a function of time for the case of a Hertzian contact pressure, with the nucleation of squeeze-out in the center of the contact area and circular growth.

Finally, we analyze analytically and via simulations the effect of normal stress variations. In a very recent paper Mukhopadhyay et al. ${ }^{25}$ have used fluorescence correlation spectroscopy to study diffusion in molecularly thin confined layers of OMCTS in a surface force apparatus (SFA) experiment. Spatially resolved measurements showed that translational diffusion slows exponentially as the probe position is moved from the edges of the contact, in the radial direction, toward the center. Assuming a Hertzian contact, the decrease in diffusivity is naturally associated with the increase in the normal pressure. Based on the Einstein relation, one may be tempted to assume that the microscopic sliding friction, $\bar{\eta}$, which enters in the squeeze-out dynamics (see below), will have a similar pressure dependence as the (inverse of the) diffusivity, suggesting that friction reflects a disproportionate contribution from those more sluggish molecules that reside near the center of a contact zone. However, we show below that this assumption leads to a squeeze-out dynamics in complete disagreement with the experiments of reported in Sec. II. One tentative explanation for this puzzling result is that the nature of the lubrication film changes during squeeze-out, from a solidlike state before squeeze-out to a liquidlike state during squeeze-out (and most likely also during sliding).

This paper is organized as follows: Section II presents experimental results. The hydrodynamic squeeze-out theory is briefly described in Sec. III and applied to the case of Hertzian contact pressure distribution. We also discuss the details of the numerical method and simulation. In Sec. IV we present numerical results and compare with the new experimental data for OMCTS. In Sec. V we discuss the experiments of Mukhopadhyay et al., and present analytical and numerical results for squeeze-out when the sliding friction $\bar{\eta}$ depends exponentially on the perpendicular pressure (which is assumed to be of the Hertzian form). Section VI present the summary and conclusions.

\section{EXPERIMENTAL RESULTS}

We observed the squeeze-out of thin films of OMCTS in an SFA geometry upon pressing two confining mica surfaces together with continuously increasing load. The experiment is similar to previous drainage experiments; ${ }^{26}$ however, the optical setup of the instrument was modified, such that we obtained two-dimensional images of the liquid layer. ${ }^{19}$ The transmitted intensity is converted to thickness using wellestablished techniques for the optical properties of multilayers. $^{27}$ 


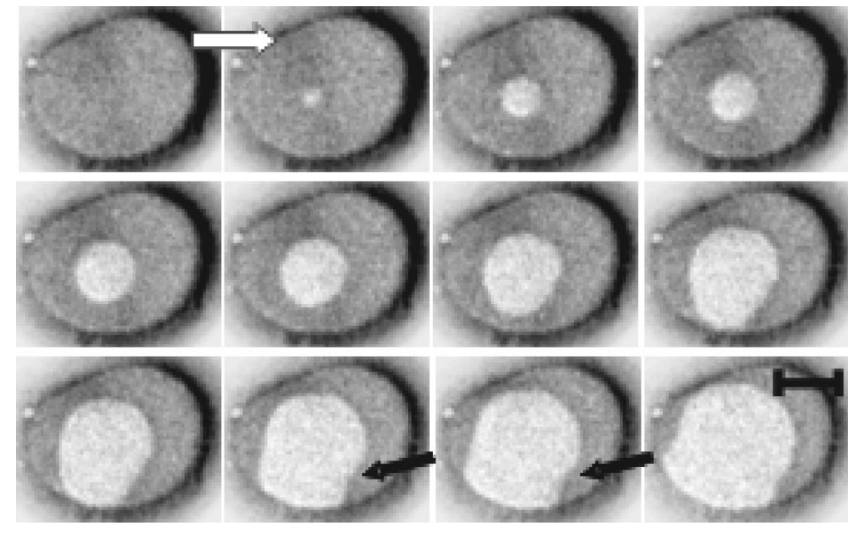

FIG. 2. Experimental snapshots of the contact area during the $n=3 \rightarrow 2$ layering transition (time between subsequent images: 0.1 second. Scale bar: $25 \mu \mathrm{m})$. The initial gray level corresponds to a film thickness of $n=3$ monolayers, and the brighter final one to $n=2$. Black arrows points to segments of the boundary line with negative curvature formed close to the edge of the contact zone. The white arrow indicates the direction of time.

Like in Ref. 22, we found that the average film thickness $h$ first decreases continuously, as expected for a bulk liquid. Below a thickness of $\sim 5 \mathrm{~nm}$, however, $h$ decreases in a series of discrete steps, each corresponding to $\Delta h=(0.95$ $\pm 0.1) \mathrm{nm}$, i.e., the diameter of one OMCTS molecule. If the contact area is sufficiently large, we can follow the evolution of $h$ as a function of time and of position within the contact area. Figure 2 shows a series of consecutive snapshot images taken during the transition from $n=3$ to $n=2$. It shows that the (brighter) $n=2$ island first appears close to the center of the contact area and then spreads across the whole contact area within about 3 seconds. While the boundary line is approximately circular initially, it deforms slightly as it approaches the edge of the contact area (second row). At this time, some sections of the boundary line assume a negative curvature (see the black arrows).

From Fig. 2, we determined also the effective radius $r(t)=[A(t) / \pi]^{1 / 2}$ of the $(n-1)$ island. For the sake of comparison to our analytical model of the layering transition (see below), it is useful to normalize $r(t)$ by the radius $R$ $=\left(A_{0} / \pi\right)^{1 / 2}$ of the contact area and plot it versus the time $t$ normalized by the squeeze-out time $\tau_{\exp }$ [cf. Eqs. (5), (9) in Sec. III below, where we also discuss the procedure of calculating it]. The result is plotted as open symbols in Fig. 5.

\section{THEORY}

We focus on the evolution of the boundary line separating the $n$ and $n-1$ regions during the layering transition $n$ $\rightarrow n-1$ for 2D-liquidlike films, when the nucleation of the layering transition occurs either in the center or off-center. Since the lubrication film is assumed to be in a 2D-liquidlike state, the basic equations of motion for the lubrication film are the continuity equation and the (generalized) Navier-Stokes equation for the 2D-velocity field $\mathbf{v}(\mathbf{x}, t)$ (we assume an incompressible 2D fluid). ${ }^{1,12}$

$$
\nabla \cdot \mathbf{v}=0
$$

$$
\frac{\partial \mathbf{v}}{\partial t}+\mathbf{v} \cdot \nabla \mathbf{v}=-\frac{1}{m n_{\mathrm{a}}} \nabla p+\nu \nabla^{2} \mathbf{v}-\bar{\eta} \mathbf{v},
$$

where $p$ is the $2 \mathrm{D}$ pressure, $\nu$ the $2 \mathrm{D}$-kinematic viscosity, and $m n_{\mathrm{a}}$ is the mass density. The last term in (2) describes the "drag force" from the solid walls acting on the fluid.

The contact area between the two solids surfaces is taken to have circular shape with radius $R$. Assume first that the initial nucleation occurs at the center of the contact. If we neglect the instability effect, ${ }^{13}$ then by symmetry the interfacial line between the squeezed and nonsqueezed area has a circular shape of radius $r(t)$. Let $p_{1}(r)$ be the 2D pressure at the (inner) moving boundary line and $p_{0}$ the spreading pressure at the (outer) boundary of the contact (at $r=R$ ). From the equations above, one can show that ${ }^{12}$

$$
\frac{d A(t)}{d t} \ln \left[\frac{A(t)}{A_{0}}\right]=-\frac{4 \pi\left(p_{1}-p_{0}\right)}{m n_{a} \bar{\eta}},
$$

where the squeezed-out area $A(t)=\pi r^{2}$ and the total contact area is $A_{0}=\pi R^{2}$. In Refs. 12 and 24, we have shown that $p_{1}=p_{0}+P(r) a$, where $P(r)$ is the normal stress acting in the contact area (we assume circular symmetry), and $a$ is the width of the layer, typically of order $1 \mathrm{~nm}$. If we assume constant normal stress $P_{0}$, then $p_{1}=p_{0}+P_{0} a$ is position independent, and ${ }^{12}$

$$
\frac{A(t)}{A_{0}}\left(\ln \left[\frac{A(t)}{A_{0}}\right]-1\right)=-\frac{t}{\tau},
$$

where $\tau$ is the time for complete squeeze-out

$$
\tau=\frac{m n_{a} \bar{\eta} A_{0}}{4 \pi P_{0} a} .
$$

Introducing $\widetilde{A}=A / A_{0}$ and $\tilde{t}=t / \tau$, Eq. (4) transforms to

$$
\widetilde{A}(\widetilde{t})(\ln \widetilde{A}(\widetilde{t})-1)=-\tilde{t} .
$$

Under most normal circumstances, a Hertzian contact pressure distribution is a much better approximation, leading to squeeze dynamics in good agreement to experiments..$^{23,24}$ The Hertzian pressure is

$$
P(r)=\frac{3}{2} P_{0}\left(1-\frac{r^{2}}{R^{2}}\right)^{1 / 2} .
$$

Combining Eqs. (3) and (7), we get (in reduced units)

$$
\frac{d \tilde{A}}{d \tilde{t}} \ln \tilde{A}=-\frac{3}{2} \sqrt{1-\widetilde{A}} .
$$

Equation (8) is easy to integrate, yielding

$$
\tilde{t}=\frac{4}{3}\left[\sqrt{1-\widetilde{A}}(\ln \tilde{A}-2)+\ln \left(\frac{1+\sqrt{1-\tilde{A}}}{1-\sqrt{1-\tilde{A}}}\right)+2-\ln 4\right] .
$$

On the other hand, if the squeeze starts off-center the symmetry properties assumed above are no longer valid, and one has to turn to numerical calculations. In that case we have shown ${ }^{23,24}$ that the equations of motion can be transformed to a simpler form. Thus, using dimensional arguments, Eq. (2) can be simplified to 


$$
\nabla p+m n_{a} \bar{\eta} \mathbf{v}=0 .
$$

This approximation assumes that the flow field is able to rearrange itself much faster than the interfacial line motion. From (10) it follows that

$$
\mathbf{v}=\nabla \phi,
$$

where

$$
\phi=-p / m n_{a} \bar{\eta} .
$$

The continuity equation (1) then gives

$$
\nabla^{2} \phi=0,
$$

which is a convenient starting point for numerical treatment. Equation (13) differs from Eq. (6) or (9) such that the explicit time dependence is absent. It is a quasistatic approximation, assuming that the flow field rearranges on a much faster time scale than the motion of the interface line separating the squeezed and nonsqueezed areas. Our numerical approach to this moving-boundary problem has been discussed in Refs. 23 and 24. It is a kinetic Monte Carlo (MC) scheme where at each step the flow field is recalculated and used for growing the squeezed area. In particular, applying this numerical scheme to centrosymmetriclike conditions, yields (with a reasonable choice of physical and numerical conditions) results in very good agreement with Eqs. (6) or (9). In Refs. 23 and 24 we have also discussed the case of position-dependent friction to account for pinning of fluid patches, along with a numerical scheme for simulating this continuum mechanics problem.

Another important feature of the theoretical treatment is the generic nature of our predictions. Given the underlying assumption that the expelled fluid is in a 2D liquidlike state, any squeeze process can be scaled to dimensionless area and time variables $\left(\tilde{A}=A / A_{0}, \tilde{t}=t / \tau\right)$ for which the dynamics is universal. Different squeeze processes, such as various $n$ $\rightarrow n-1$ transitions or even different lubricants, are associated with different values of physical parameters (such as density microscopic friction $\bar{\eta}$ ), therefore different scaling factors. $\tau$ is a function of the microscopic fluid parameters and of the experimental setup. $A_{0}$ is only a function of the experimental setup. Scaling experimental (or simulations) results to reduced units is simple and automatic if we assume that at short enough times the squeeze process evolves in centrosymmetric fashion, following Eq. (9). $A_{0}$ is assumed to be known experimentally, and thus we evaluate the righthand side of Eq. (9). For short times it is a linear function with slope $1 / \tau$. This procedure proved to be very accurate.

In what follows, however, we study only universal aspects of the squeeze process, and compare our calculations to the experimental results discussed in Sec. II for the $3 \rightarrow 2$ transition.

\section{RESULTS AND DISCUSSION}

We start with analyzing the two analytical solutions of the idealized centrosymmetric squeeze. Figure 3 shows the analytical solutions to the centrosymmetric case both for a Hertzian squeezing pressure [continuous line, given by Eq. (9)] and for a constant pressure [dash-dotted line, given by
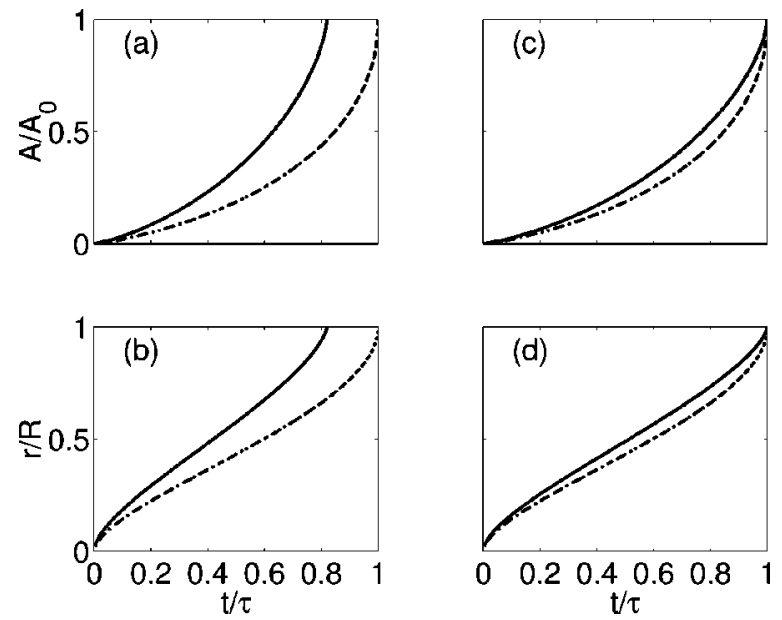

FIG. 3. Analytical solutions to the centrosymmetric squeeze problem, for Hertzian normal stress (continuous line) and constant stress profile (dashdotted line). (a) Reduced area and (b) reduced radii $(\widetilde{r}=\sqrt{\widetilde{A}})$ of the squeezed circle vs reduced time. (c) and (d) show the same as (a) and (b) but now with the Hertzian curves scaled such that the total squeeze time would be the same as in the constant stress case (see the text).

(6)]. We show the variation of (a) the squeezed area and (b) the squeeze radii versus time. It is clearly seen that the Hertzian profile predicts faster squeeze-out (by roughly 20\%), but qualitatively both solutions are similar. In Figs. 3(c) and 3(d) we have scaled the time so that the squeeze-out time would be unity in all cases; in this case it is hard to distinguish from the qualitative line shapes between the two cases.

We have also performed computer simulations of the squeeze-out process, using the methods discussed above and in Ref. 24. In the present experiments (Fig. 2, see also Ref. 21) the squeeze process started close to the center, and the interfacial line was smooth on the length scale of the contact radius $R$. Thus, no fluid was trapped in the contact, and the propagation was nearly centrosymmetric. This is in contrast to previous systems studied in Ref. 19, where the line tension was low enough to produce a rough boundary line, and trapped fluid islands. The value of line tension, however, is related to the effective elasticity of the mica substrates. ${ }^{13}$ The latter was exceptionally low in Ref. 19, because the mica thickness was $<500 \mathrm{~nm}$ then, whereas it is $2.5 \mu \mathrm{m}$ in the present experiments. For our simulations, we chose a combination of parameters for the line tension and the smoothening dynamics ${ }^{24}$ such as to reproduce the smooth boundary lines shown in Fig. 2.

In our computer simulations two cases were considered, one in which the squeeze-out started in the center of the contact area, and another which started slightly off-center $(r=0.15 R)$. Figure 4 shows snapshots of the two systems. Note that the roughness of the boundary line is of the right length scale, and that (as expected) the boundary line of the off-center case propagates faster toward the nearest edge of the contact area, while the centered case evolves in a quasisymmetrical fashion.

As stated above, we have used in the simulations a kinetic Monte Carlo (MC) scheme, where the MC time scale is linearly related to the physical time scale: $\tilde{t}=\alpha t_{\mathrm{MC}}$ (note that the MC time scale itself is nonlinearly related to the number 


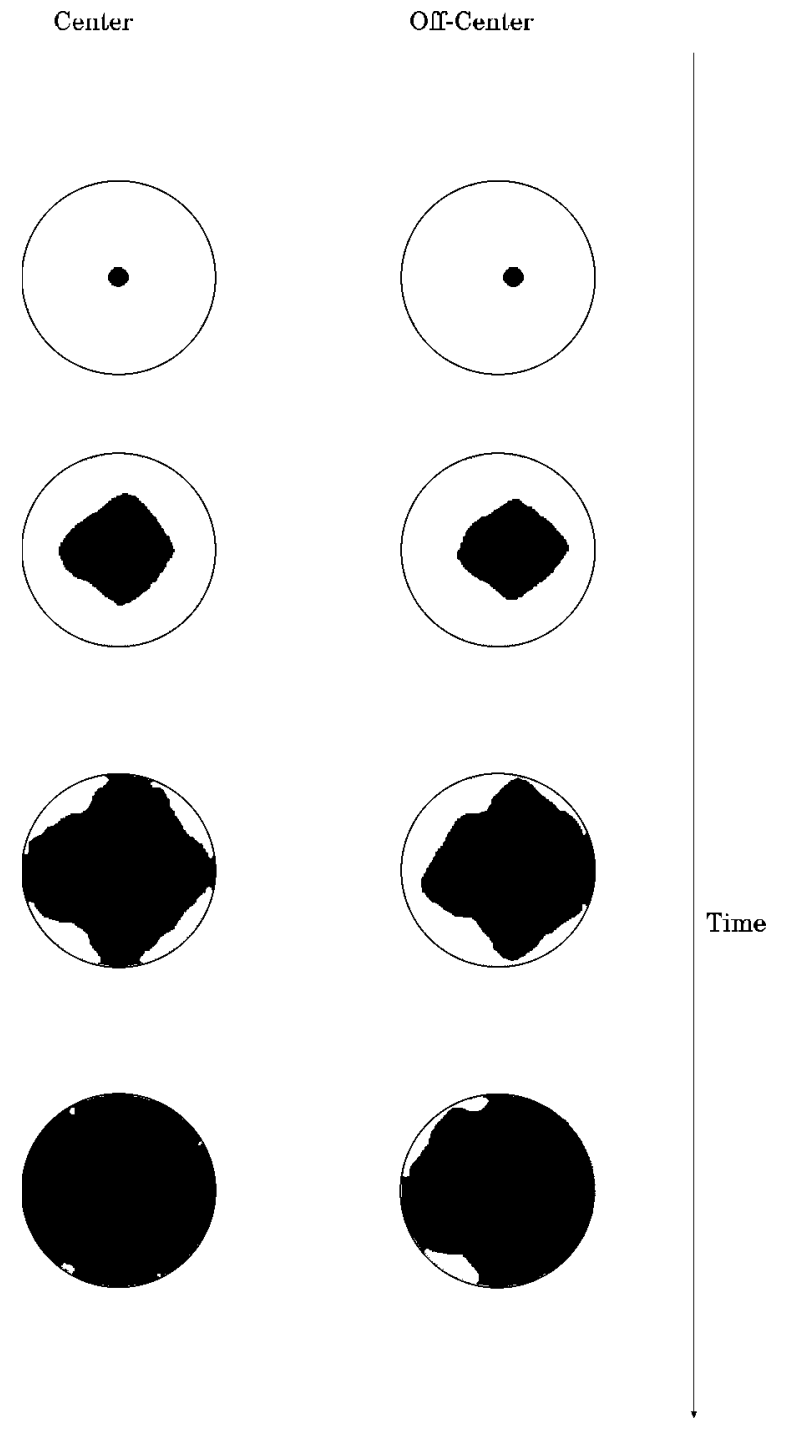

FIG. 4. Simulation snapshots of squeeze dynamics. Initial (small) squeezed circular zone is assumed located in the center of the contact (left figures) and slightly off-center $(r=0.15 R$, right figures). The black area indicates squeezed zones and white the fluid ones. The overall shape of the contact area is circular. Time arrow is also indicated.

of MC steps in the simulation; see Ref. 24 for more details). In order to properly scale the results we plot the left-hand side of Eq. (9) (with $\widetilde{A}$ data from the simulations) versus $t_{\mathrm{MC}}$ to find the scaling factor $\alpha$.

Figure 5 shows the time evolution of the effective radius of the squeezed area. We show both simulations results and the analytical result [from Fig. 3(d)]. The effective radius is defined by $\widetilde{r}=\widetilde{A}^{1 / 2}$. The agreement between the analytical formula and the simulations is excellent; the results differ only toward the end of the squeeze-out process $(0.8<t / \tau$ $<1$ ), where the circular symmetry is completely lost. More remarkable is the agreement between the off-center simulations and the analytical solution, which is centrosymmetric. It is easy to qualitatively understand why the squeeze time is prolonged when the symmetry is broken: the flow lines no longer point only in the radial direction but a more complex flow pattern occurs in the 2D fluid such that the net flow out of the boundaries of the contact region is slower. Similar

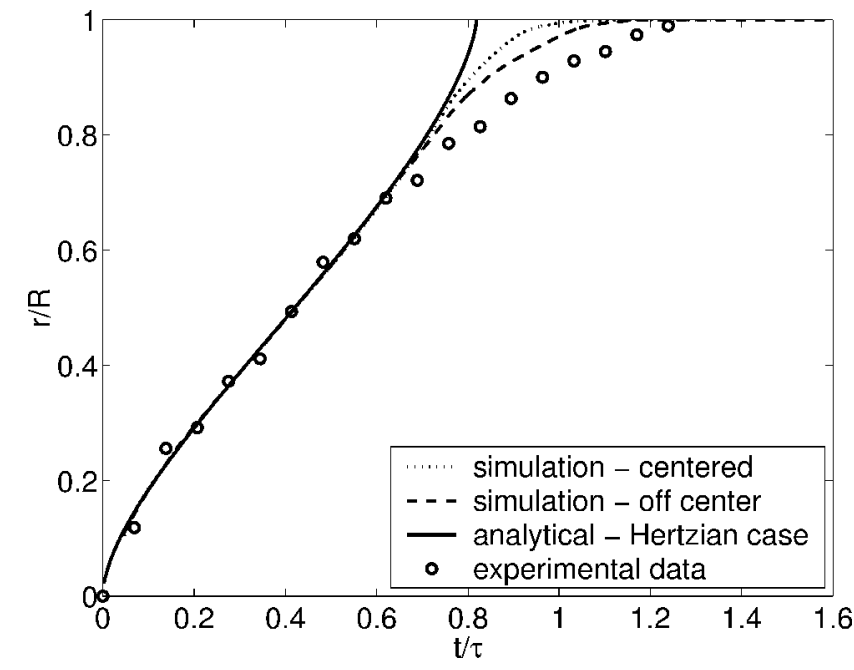

FIG. 5. Effective squeeze radii from experiments simulations and analytical calculations. All calculations used Hertz stress profile.

effects were observed in previous publications ${ }^{23,24}$ where different time scales in the squeeze-out process were reported. In particular, we have shown there that the squeeze rate decreases as the boundary line becomes more complex. Figure 5 shows that the relation between the effective radius $r$ and the squeeze time $\tau$ is rather insensitive both to where the squeeze-out nucleates and to the detailed form of the squeezed-out area. This is a very important result since in practical applications the squeeze-out will never start exactly in the center of the contact area, and the squeezed-out region will never be perfectly circular.

The agreement between simulations results and the experimental data during most of the squeeze process, as shown in Fig. 5, is quite remarkable. Similarly remarkable is the qualitative agreement at long times, when compared to the qualitative deviation of the analytical result that is based on the circular symmetry assumption. This emphasizes the deviation from circular symmetry at long times, and in particular the slowdown of the squeeze-out rate as compared to that predicted from the circular symmetry model, as the main source of disagreement between theory and experiment. The exact amount of slowdown in not so easy to predict, as it likely depends on the line energy which we do not know accurately, and also because it will depend on statistical (e.g., thermal) fluctuations in the system. It may possibly even vary from one experimental run to another (this has not been tested experimentally yet).

Finally, let us compare the theory with the experiments. From Fig. 2, we saw that circular symmetry approximately prevailed up to the seventh or the eighth image. Since the analytical solution is based on circular symmtery we can expect it to hold only up to this time. The calculation of the experimental squeeze-time parameter $\tau_{\exp }$ [cf. Eq. (5)] is the result of a linear fit to Eq. (9), as discussed in Sec. III. As shown in Fig. 5, the agreement between the experimental data and the theory is quite good, and we conclude that the OMCTS film most likely is in a 2D liquidlike state, at least during squeeze-out. This assumption is further supported by recent computer simulations of Persson et al. ${ }^{18}$ 


\section{POSITION-DEPENDENT RADIAL FRICTION}

In a very recent paper Mukhopadhyay et al. ${ }^{25}$ have used fluorescence correlation spectroscopy to study diffusion in molecularly thin confined layers of OMCTS. Spatially resolved measurements showed that translational diffusion slows exponentially with increasing perpendicular pressure from the edges of a Hertzian contact toward the center, suggesting that friction reflects a disproportionate contribution from those more sluggish molecules that reside near the center of a contact zone. Based on the Einstein relation, one may be tempted to assume that the sliding friction, $\bar{\eta}$, which enters in the squeeze-out dynamics, will have a similar pressure dependence as the (inverse of the) diffusivity. Note that the validity of Einstein relation under our conditions cannot be taken for granted, although it is consistent with our assumption that the lubricant is in a liquidlike state during squeezeout. In any case, we show below that this assumption leads to a squeeze-out dynamics in complete disagreement with the experiments shown in Fig. 2. One tentative explanation for this puzzling result is that the nature of the lubrication film changes at the onset of squeeze-out, from a solidlike state before squeeze-out to a liquidlike state during squeeze-out (and most likely also during sliding).

In this section we analyze analytically and numerically the implications of assuming a position-dependent friction. Mukhopadhyay observed that the diffusivity in a threemonolayer-thick OMCTS film depended exponentially on the perpendicular pressure, $D \sim \exp [-\alpha P(r)]$, where $\alpha$ is an empirical exponent and $P(r)$ is the normal stress which we will take to be of the Hertzian form. In accordance with the Einstein relation, we will assume $\bar{\eta} \sim 1 / D$ so that $\bar{\eta}(r)$ $\sim \exp [\alpha P(r)]$. Thus, if $\eta_{0}$ denote the average friction

$$
\bar{\eta}(r)=\frac{\eta_{0} \exp [\alpha P(r)]}{\left(2 / R^{2}\right) \int_{0}^{R} r \exp [\alpha P(r)] d r} .
$$

Putting in the Hertzian pressure profile [Eq. (7)] and turning to the reduced length units, we obtain

$$
\begin{aligned}
& \bar{\eta}(\widetilde{r})=\eta_{0} K \exp \left[\lambda \sqrt{1-\widetilde{r}^{2}}\right], \\
& K=\frac{\lambda^{2} / 2}{1-e^{\lambda}+\lambda e^{\lambda}},
\end{aligned}
$$

with $\lambda=(3 / 2) \alpha P_{0}$. In Fig. 6 we show the friction $\bar{\eta}(r)$ for a few different $\lambda$. From the diffusion experiments reported on in Ref. 25 we deduce $\lambda=8.6$. Let us now study the implications of using (15) for the simplified centrosymmetric squeeze. In the quasistatic approximation, we have

$$
\frac{\partial p}{\partial r}=-m n_{0} \bar{\eta} v
$$

where

$$
v(r, t)=\frac{B(t)}{r},
$$

where $B(t)$ is some auxiliary unknown function of time only. Thus

$$
\frac{\partial p}{\partial r}=-m n_{0} \bar{\eta}(r) \frac{B(t)}{r} .
$$

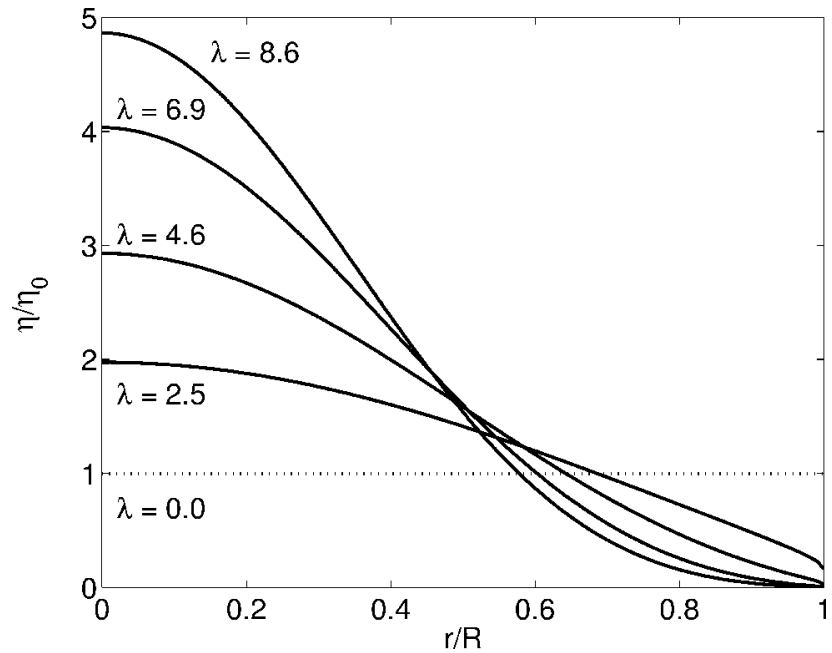

FIG. 6. Position-dependent radial friction for various values of the exponential parameter $\lambda$.

Integrating from $r=r_{1}(t)$ to $r=R$ gives

$$
p_{1}-p_{0}=m n_{0} B(t) \int_{r_{1}}^{R} d r \frac{\bar{\eta}(r)}{r} .
$$

Using Eq. (18) with $r=r_{1}(t)$ and $v\left(r_{1}, t\right)=\dot{r}_{1}$, we get

$$
B(t)=r_{1} \dot{r}_{1},
$$

and

$$
p_{1}-p_{0}=m n_{0} r_{1} \dot{r}_{1} \int_{r_{1}}^{R} d r \frac{\bar{\eta}(r)}{r},
$$

or

$$
\int_{0}^{r_{1}} d r^{\prime} \frac{r^{\prime}}{p_{1}\left(r^{\prime}\right)-p_{0}} \int_{r^{\prime}}^{R} d r^{\prime \prime} \frac{\bar{\eta}\left(r^{\prime \prime}\right)}{r^{\prime \prime}}=t .
$$

In the present case this gives, with $x=r^{\prime} / R$ and $y=r^{\prime \prime} / R$

$$
\int_{0}^{r / R} d x \int_{x}^{1} d y \frac{x}{y} \frac{\exp \left[\lambda\left(1-y^{2}\right)^{1 / 2}\right]}{\left(1-x^{2}\right)^{1 / 2}}=\frac{3 t}{8 K \tau} .
$$

In the limit $\lambda \rightarrow 0$ we get $K \rightarrow 1$ and Eq. (24) reduces to

$$
\int_{0}^{r / R} d x \frac{x \log x}{\left(1-x^{2}\right)^{1 / 2}}=-\frac{3 t}{8 \tau} .
$$

Since $\widetilde{A}=(r / R)^{2}$, this equation is equivalent to Eq. (9).

In Fig. 7 we show the dependence of the squeeze radius on time for different values of the parameter $\lambda$. From the diffusion experiments in Ref. 25 we deduce $\lambda \approx 8.6$. Note that when $\lambda$ increases, the squeeze-out is slower at the early stages where the friction $\bar{\eta}>\eta_{0}$ up to $\widetilde{r}_{1} \approx 0.7$, where it becomes significantly faster. However, the complete squeezeout time is rather insensitive to the value of $\lambda$, and even for very large $\lambda$ it is only $\sim 15 \%$ smaller than for $\lambda=0$. The reduction in the squeeze-out time saturates as we increase $\lambda$.

We have also performed computer simulations with the position-dependent friction given by Eq. (15), for various values of the $\lambda$ parameter, ranging from zero (positionindependent friction) to 8.6, which is similar to the experimental value deduced from the diffusivity data. ${ }^{25}$ The 


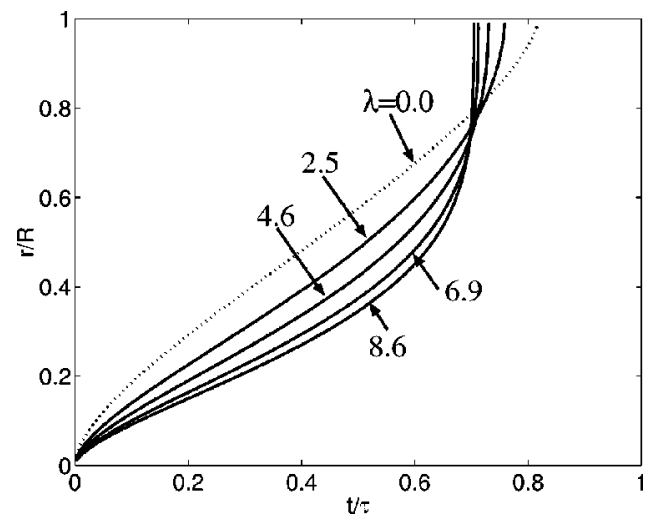

FIG. 7. Squeeze radius vs time, for different values of $\lambda$. Analytical calculations based on Eq. (24).

results are summarized in Fig. 8. Figure 8 shows that for short times (up to $\tilde{t} \approx 0.7$ ) the evolution of the squeezed area is very similar to the ideal analytical case discussed above (Fig. 7). However, for longer times the simulation results strongly diverge from the prediction of centrosymmetric squeeze, and the squeeze-out time becomes many orders of magnitude longer (which we could not follow) than what follows by assuming centrosymmetric squeeze. This effect is due to the breaking of the circular symmetry, and to the formation of (temporary) trapped fluid islands (see Fig. 9 for an example). In Ref. 24 we have already shown under

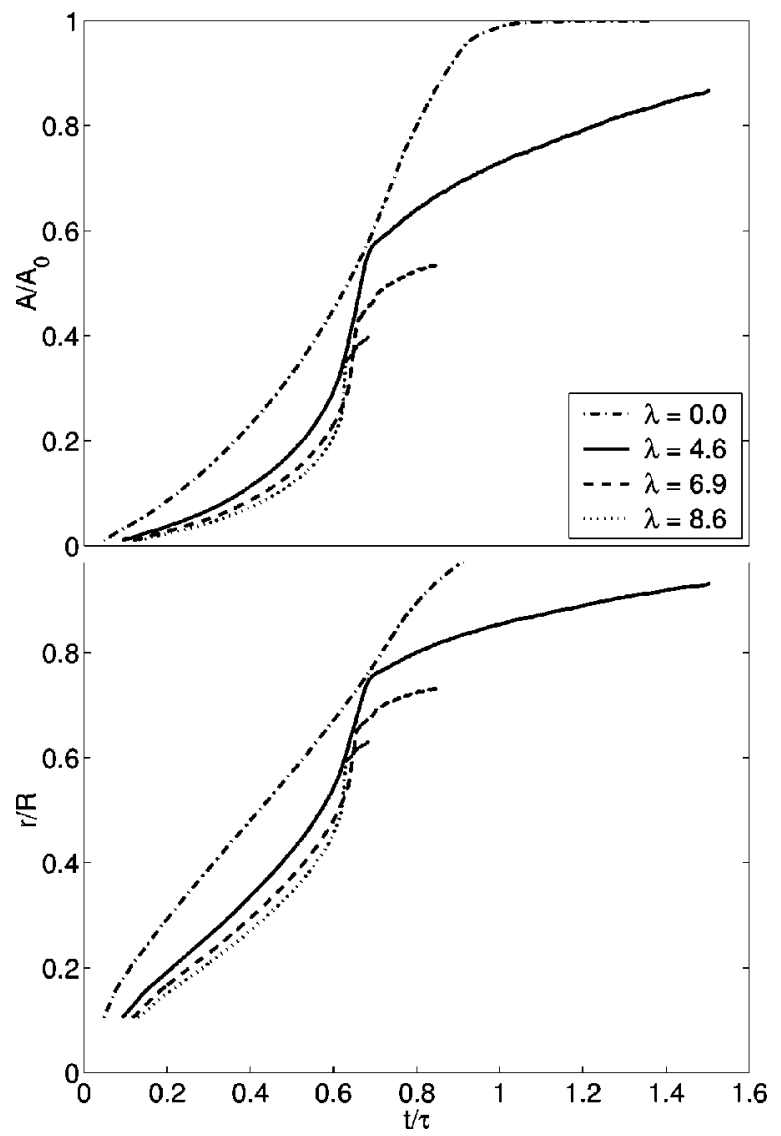

FIG. 8. Simulations with position-dependent friction.

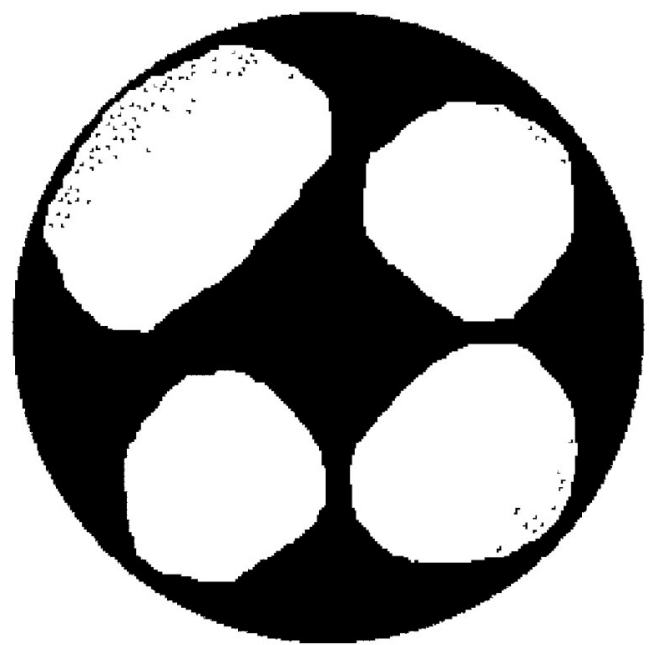

FIG. 9. A snapshot picture of the squeeze pattern we obtained when we stopped the simulation $(\lambda=8.6)$.

slightly different conditions that trapped fluid pockets are squeezed out on a much longer time scale than the initial bulk of the fluid.

It is clear that, if one assumes that the sliding friction is related to the diffusivity via the Einstein relation, the squeeze-out dynamics predicted with a position-dependent friction is in complete disagreement with the diffusivity experiments of Mukhopadhyay et al. Our analytical results and computer simulations shows that only a very weak $r$ dependence of $\bar{\eta}$ is possible in order to avoid disagreement with the measurements of the layer expulsion dynamics (Fig. 2). We tentatively attribute this observation to a phase transformation of the lubrication film from a solidlike state before squeeze-out to a liquidlike state during squeeze-out. In any case the observed discrepancy seems to be very fundamental, and requires further investigation.

Finally, we note that the pressure index $\alpha$ observed by Mukhopadhyay is much larger than what has been observed from the pressure dependence of bulk liquids. For bulk liquids the viscosity usually depends exponentially on the pressure, $\sim e^{\alpha P}$ with $\alpha \approx 10^{-8} \mathrm{~Pa}^{-1}$. In contrast, the pressure index of the diffusivity observed by Mukhopadhyay is of the order $\alpha \approx 10^{-6} \mathrm{~Pa}^{-1}$. If the pressure index of the confined film would have been as small as observed for bulk liquids, no dependence of the diffusivity on the Hertzian pressure would be expected in the measurements reported on in Ref. 25 .

\section{SUMMARY}

The continuum mechanics theory of squeeze-out has been solved analytically for a Hertzian normal stress and centrosymmetric squeeze-out. For the off-center nucleation of squeeze-out, we have presented numerical results based on the kinetic Monte Carlo method. The theoretical results have been compared with recent measurements that will be described in more details elsewhere. ${ }^{21}$ We find good agreement between theory and experiment which suggests that the assumption of a 2D liquidlike lubrication films is a good 
approximation, at least for the $n=3 \rightarrow 2$ transition, to which the experimental data refer. However, it may very well be that the $n=2 \rightarrow 1$ and $n=1 \rightarrow 0$ squeeze-out transitions are more solidlike, perhaps involving plastic deformation within the 2D-lubrication film. So far, we have not been able to squeeze-out the last OMCTS monolayer, but intensive experimental efforts are underway to probe the nature of this transitions.

We have studied the influence of a position-dependent friction and shown that the resulting squeeze-out dynamics is in complete disagreement with the diffusivity experiments of Mukhopadhyay et al. Our analytical results and computer simulations shows that only a very weak $r$ dependence of $\bar{\eta}$ is possible in order to avoid disagreement with our measurements, while the diffusivity data of Mukhopadhyay et al. indicate a very strong $r$ dependence of the diffusivity. We tentatively attribute this discrepancy to a phase transformation of the lubrication film from a solidlike state before squeezeout to a liquidlike state during squeeze-out. The observed discrepancy requires much more investigation.

\section{ACKNOWLEDGMENTS}

Two of the authors (B.P. and A.N.) thank BMBF for a grant related to the German-Israeli Project Cooperation "Novel Tribological Strategies from the Nano-to MesoScales." A.N. thanks the Israel Science Foundation for partial support of this work (Grant No. 248/99). F.M. and T.B. acknowledge funding by the German Science Foundation within the priority program "Wetting and Structure Formation at Interfaces."
${ }^{1}$ B.N.J. Persson, Sliding Friction: Physical Principles and Applications, 2nd ed. (Springer, Heidelberg, 2000).

${ }^{2}$ B.N.J. Persson, Surf. Sci. Rep. 33, 83 (1999).

${ }^{3}$ J. Krim, Sci. Am. 275, 74 (1996).

${ }^{4}$ J.N. Israelachvili, Intermolecular and Surface Forces (Academic, London, 1995).

${ }^{5}$ M.L. Gee, P.M. McGuiggan, and J.N. Israelachvili, J. Chem. Phys. 93, 1895 (1990).

${ }^{6}$ J. Gao, W. D. Luedtke, and Uzi Landman, Phys. Rev. Lett. 79, 705 (1997).

${ }^{7}$ H. Tamura, M. Yoshida, K. Kusakabe, C. Young-Mo, R. Miura, M. Kubo, K. Teraishi, A. Chatterjee, and A. Miyamoto, Langmuir 15, 7816 (1999).

${ }^{8}$ A. Levent Demirel and S. Granick, Phys. Rev. Lett. 77, 2261 (1996).

${ }^{9}$ A. Levent Demirel and S. Granick, J. Chem. Phys. 106, 6889 (1998).

${ }^{10}$ J. Klein and E. Kumacheva, Science 269, 816 (1995).

${ }^{11}$ E. Kumacheva and J. Klein, J. Chem. Phys. 108, 7010 (1998).

${ }^{12}$ B.N.J. Persson and E. Tosatti, Phys. Rev. B 50, 5590 (1994).

${ }^{13}$ B.N.J. Persson, Chem. Phys. Lett. 324, 231 (2000). See also Fractals and Disordered Systems, edited by A. Bunde and S. Havlin (Springer, New York, 1991); J.S. Langer, Phys. Rev. A 36, 3350 (1987); E. Brener, Phys. Rev. Lett. 71, 3653 (1993).

${ }^{14}$ U. Raviv, P. Laurat, and J. Klein, Nature (London) 413, 51 (2001).

${ }^{15}$ A. Dhinojwala and S. Granick, J. Am. Chem. Soc. 119, 241 (1997).

${ }^{16}$ B.N.J. Persson and P. Ballone, Solid State Commun. 115, 599 (2000).

${ }^{17}$ B.N.J. Persson and P. Ballone, J. Chem. Phys. 112, 9524 (2000).

${ }^{18}$ B.N.J. Persson, V.N. Samoilov, S. Zilberman, and A. Nitzan, J. Chem. Phys. 117, 3897 (2002).

${ }^{19}$ F. Mugele and M. Slameron, Phys. Rev. Lett. 84, 5796 (2000).

${ }^{20}$ F. Mugele and M. Salmeron, J. Chem. Phys. 114, 1831 (2001).

${ }^{21}$ T. Becker and F. Mugele, J. Phys.: Condens. Matter 15, 321 (2003); T. Becker, H. Herminghaus, and F. Mugele (unpublished).

${ }^{22}$ See Sec. 8.7 in Ref. 1. See also D. Forster, D.R. Nelson, and M.J. Stephen, Phys. Rev. A 16, 732 (1977); S. Ramaswamy and G. Mazenko, ibid. 26, 1735 (1982)

${ }^{23}$ S. Zilberman, B.N.J. Persson, A. Nitzan, F. Mugele, and M. Salmeron, Phys. Rev. E 63, 055103 (2001).

${ }^{24}$ S. Zilberman, B.N.J. Persson, and A. Nitzan, J. Chem. Phys. 115, 11268 (2001).

${ }^{25}$ A. Mukhopadhyay, J. Zhao, S.C. Bae, and S. Granick, Phys. Rev. Lett. 89, 136103 (2002).

${ }^{26}$ D.Y.C. Chan and R.G. Horn, J. Chem. Phys. 83, 5311 (1985).

${ }^{27}$ F. Mugele, T. Becker, A. Klingner, and M. Salmeron, Colloids Surf., A 206, 105 (2002). 